\title{
Commentary on Gilad Rabinovitch's “Unplayed Galant Melodies, the Ubiquity of the Rarest Interval, and the Heyday of the Major Mode."
}

\author{
DAVID CLAMPITT \\ Ohio State University [1]
}

\begin{abstract}
This is a commentary accompanying Rabinovitch (2019), situating the research within some previous work on scale theory.
\end{abstract}

Submitted 2019 October 20; accepted 2019 October 20

Published 2020 July 6; https://doi.org/10.18061/emr.v14i3-4.7571

KEYWORDS: Galant schemata; scale theory

THIS article by Rabinovitch (2019) tests the hypothesis that the rare intervals of the diatonic template (tritone and semitone) are emphasized in Gjerdingen's (2007) schemata in usage, in terms of the essential scale degrees of the schemata, what Rabinovitch calls their skeletons. This is a reasonable guess, when one considers the primal schema, the Meyer, ${ }^{\wedge} 1-^{\wedge} 7 . .^{\wedge} 4-^{\wedge} 3$, which embraces the two semitones of the diatonic set, and typically accompanies $\wedge 4$ with the diminished fifth as a vertical interval. Similarly, the Fenaroli, ${ }^{\wedge} 4-^{\wedge} 3-$ $\wedge 7-\wedge 1$. Indeed, one could collate the schemata in their abstract forms (e.g., the "schema prototypes" collected in Gjerdingen, 2007, Appendix A) and tabulate the intervals between successive scale degrees in the treble, and the treble-bass verticalities, to discover the ratios of rare intervals to common intervals. This would be a worthwhile exercise although there would be questions to work out (one of them, whether to assume major mode for the schemata, is thematic in this study whose title refers to "the heyday of the major mode").

Rabinovitch, however, is interested in the schemata in their actual usage in a corpus of Galant music, and he differentiates his skeletons from the schema prototypes: "[G]alant schemata are not yet observable features" (p. 91). For methodological reasons that he presents in the article, he chooses Gjerdingen's own collection of analyzed complete movements in Music in the Galant Style. Given the striking predominance of major-mode compositions in the second half of the eighteenth century, as compared especially with the first half of the century and also with the nineteenth century (see Table 1), his decision to assume a majorkey environment is reasonable. While Gjerdingen sets the limits of the Galant style as spanning thirty years on either side of the half-century divide, 1720-1780, the fourteen movements Rabinovitch analyzes are in fact all in the major mode. Thus, the expectation is that most schema appearances will be in a major contextthose associated with beginnings and endings of pieces and interior cadences or passages in closely related dominant or subdominant keys (adopting an attitude he refers to as "hyper-local tonal identity," the attitude historically more pertinent than "later monotonal conceptions"), together with, however, minor mode "touches," usually shifts to parallel minor or tonicizations of other minor keys.

As part of his title suggests ("the ubiquity of the rarest interval"), an over-representation of the vertical tritone in this corpus is in fact realized. The perhaps more problematic question of tallying melodic semitones does not, he concludes, bear out his hypothesis (but we will see that an interpretation much more favorable to his hypothesis is indeed available).

The motivation to tabulate rare intervals is situated in relation to the "rare interval hypothesis" (Browne, 1981; Brown \& Butler, 1981; Butler, 1989). Browne (1981) departs from the observation that diatonic intervals from the semitone to the tritone have unique multiplicities, covering integers 1 to 6 (i.e., the interval vector of Forte's, 1973, set-class 7-35, corresponding to the diatonic scale, is $<254361>$ ). The rare intervals are thus the semitone and tritone, multiplicities 2 and 1, respectively. Browne explores the dichotomy of "position finding" vs. "pattern matching," where the rare intervals enable the former and the common intervals the latter. Pattern matching, a kind of self-similarity of the diatonic set, suggests a balance of sameness and difference in sub-objects of the set; an environment not too boring yet not too exciting; a reduced cognitive load in processing. Position finding, on the other hand, is the relevant 
side of the dichotomy here: the half steps, their asymmetric (maximally even) distribution within the octave, are marked by their rarity with respect to the whole steps, and the uniqueness of the tritone determines the particular diatonic set (as long as one can discern whether the interval is to be understood as a diminished fifth or an augmented fourth). That is, up to enharmonicism, a tritone and one other note of the diatonic set uniquely determine with which of the 12 diatonic collections we are dealing (see Brown \& Butler, 1981).

To the extent we are willing to conflate diatonic collections and major keys, then, position finding is tantamount to (major) key finding. Note, however, that the interval vector is a property of the pitch-class set, not only of the corresponding major scale. To the extent that one would consider the natural minor scale to be the foundation for the relative minor key, this would be the same collection. In any case, the interval vector is invariant for all of the diatonic modes. Thus, a priori, exposure to rare intervals would not be sufficient to determine the key. Rabinovitch notes: "Indeed, some of the recent or semi-recent experiments suggest that scale-degree frequencies by themselves are not sufficient tonal cues for listeners" (p. 92).

To tease this out, in the spirit of Rabinovitch's arm-chair theorizing, first observe that tritones and semitones within the diatonic are bound up together: the diminished fifth is the only diatonic fifth that encloses both semitones (STTS); the augmented fourth is the only diatonic fourth that excludes both semitones (TTT). The diminished fifth resolves to a major third by shedding two semitones: STTS $\longrightarrow$ TT; the augmented fourth resolves to a minor sixth by adding two semitones: TTT $\rightarrow$ STTTS. This is an abstract and defamiliarized way of conceptualizing what may be musically realized as the contrapuntal combinations (now assuming a major-scale ordering):

$\begin{array}{lllll}\wedge 4 & \wedge 3 & \text { or } & \wedge 7 & \wedge 1 \\ \wedge 7 & \wedge 1 & \wedge 4 & \wedge 3\end{array}$

In these progressions, all of the linear motions and all the vertical intervals are the rare specific intervals of their generic type: in each, the two melodic step intervals are the rare semitones, the initial vertical intervals are the unique versions of the fifth or fourth, the resolution vertical intervals are major third or minor sixth (rarer with respect to their generic counterparts). The assignment of scale degrees was predicated on major, but one might rather consider the tropism embodied in the progressions to motivate the assignment, privileging what we then dub scale degree ${ }^{\wedge} 1$. Rabinovitch does not name it, but he implicitly appeals to Myhill's Property (Clough \& Myerson, 1985): each non-zero (non-octave equivalent) diatonic generic interval comes in two specific sizes within the diatonic set. Thus, in the diatonic template, generic steps and sevenths are associated with a common-to-rare multiplicity ratio 5:2, generic thirds and sixths with ratio 4:3, and generic fourths and fifths with ratio 6:1.

I doubt anyone arguing in good faith ever understood the rare interval hypothesis to presume to say anything about the frequencies of intervals in usage, but the questions are of interest, and Rabinovitch cites corpus studies of melodic intervals by Temperley (of classical string quartet first violin parts; reported in a personal communication to Rabinovitch, p. 93 of "Unplayed Galant Melodies) and by Huron (of the Essen Folksong Collection; 2006), and of vertical intervals by Huron (in Bach Inventions; 1993). We have the diatonic template with its multiplicities, completely paradigmatic. By contrast, we have the frequencies of occurrence of the intervals in three corpora, partaking of usage but not syntagmatic, because there is no recognition of sequential order. The contrapuntal combinations adduced above are both paradigms and syntagms: out-of- time with respect to actual music but order-dependent within themselves. They clearly are moving closer to the combinations that are the Galant schemata, which are paradigms/syntagms that participate in the syntagmatic domain.

Rabinovitch tests his hypotheses of over-representation of rare intervals in the skeletons of schemata in the corpus against both the diatonic template and against the results of the surface frequencies in the respective corpora. For vertical tritones, the results are positive: vis-à-vis the 6.00 ratio of the template, the ratio of $\mathrm{P} 5 / \mathrm{P} 4$ to tritone appearances in Appendix A1 is 1.48; correcting for a possible bias in the treatment of Quiescenza, in Appendix A2 the ratio is 1.82; both over-representing tritones as well with respect to the Two-Part Inventions ratio of 3.42. For the semitones, it would be highly unlikely that the skeletal soprano transitions in the Galant corpus would do better than Temperley's 1.08 ratio (as he discusses, this lowalmost even - ratio is likely due to the rather high degree of chromaticism in his corpus, and considering chromatic as well as diatonic semitones in the semitone tally; I would add that works in minor would skew the results as well). Measuring against this ratio, he considers the ratio of 1.37 to disconfirm his hypothesis ("does not support the notion that skeletal transitions are favorable to semitones," p. 98). I rather think that it 
does, when compared to the template ratio of 2.5 and the ratio 2.55 in the Essen study by Huron; the latter, because of its more exclusively diatonic and major orientation, may be a more appropriate comparison, albeit Mozart and Haydn are at least partially within the Galant canon. Consider that the corpus yields 351 semitones to 483 whole tones; if it were to match the template ratio with the same number of whole tones, it would have yielded only 193 semitones.

In any case, I think a version of the rare interval hypothesis and Rabinovitch's hypotheses in terms of skeletons of Galant schemata go well together and are potentially mutually reinforcing. This is not the place to expand upon it, but the historical trajectory towards and then away from the major mode in the course of the eighteenth century and into the nineteenth he discusses, and the association of the major mode and Galant schemata that he posits, might well be explored in conjunction with recent theoretical work in scale theory.

\section{ACKNOWLEDGEMENTS}

This article was copyedited by Niels Chr. Hansen and layout edited by Diana Kayser.

\section{NOTES}

[1] Correspondence can be addressed to: Professor David Clampitt, Ohio State University, 110 Weigel Hall, 1866 College Road N, Columbus, OH 43210. clampitt.4@osu.edu.

\section{REFERENCES}

Brown, H., \& Butler, D. (1981). Diatonic trichords as minimal cue cells. In Theory Only, 5(6-7), 39-55.

Browne, R. (1981). Tonal implications of the diatonic set. In Theory Only, 5(6-7), 3-21.

Butler, D. (1989). Describing the perception of tonality in music: a critique of the tonal hierarchy theory and a proposal for a theory of intervallic rivalry. Music Perception, 6(3), 219-241. https://doi.org/10.2307/40285588

Clough, J., \& Myerson, G. (1985). Variety and multiplicity in diatonic systems. Journal of Music Theory, 29(2), 249-270. https://doi.org/10.2307/843615

Forte, A. (1973). The structure of atonal music. New Haven, CT: Yale University Press.

Gjerdingen, R. O. (2007). Music in the Galant style. New York, NY: Oxford University Press.

Huron, D. (1993). Note-onset asynchrony in JS Bach's two-part Inventions. Music Perception, 10(4), 435443. https://doi.org/10.2307/40285582

Huron, D. (2006). Sweet anticipation: Music and the psychology of expectation. Cambridge, MA: MIT Press. https://doi.org/10.7551/mitpress/6575.001.0001

Rabinovitch, G. (2019). Unplayed galant melodies, the ubiquity of the rarest interval, and the heyday of the major mode. Empirical Musicology Review, 14(3-4), 90-134. https://doi.org/10.18061/emr.v14i3-4.6070 